\title{
The Role of Self-Compassion, Dispositional Mindfulness, and Emotion Regulation in the Psychological Health of Family Carers of Older Adults
}

\author{
Ms Jenny Murfield BSc(Hons) ${ }^{\mathrm{a}, \mathrm{b}^{*}}$, Professor Wendy Moyle $\mathrm{PhD}^{\mathrm{a}, \mathrm{b}}$, \\ Professor Analise O'Donovan $\mathrm{PhD},{ }^{\mathrm{c}}$ and Professor Robert S Ware $\mathrm{PhD}^{\mathrm{a}, \mathrm{d}}$

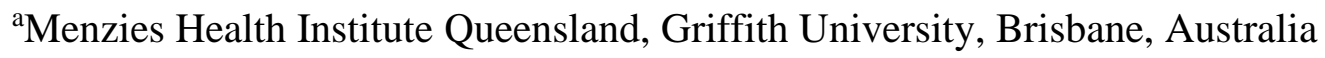 \\ ${ }^{\mathrm{b}}$ School of Nursing and Midwifery, Griffith University, Brisbane, Australia \\ ${ }^{\mathrm{c}}$ Griffith Health Group, Griffith University, Brisbane, Australia \\ ${ }^{\mathrm{d} S c h o o l ~ o f ~ M e d i c i n e, ~ G r i f f i t h ~ U n i v e r s i t y, ~ B r i s b a n e, ~ A u s t r a l i a ~}$
}

Jenny Murfield: https://orcid.org/0000-0001-9595-4242

Wendy Moyle: https://orcid.org/0000-0003-3004-9019

Analise O’Donovan: https://orcid.org/0000-0002-0776-0956

Robert Ware: https://orcid.org/0000-0002-6129-6736

*Correspondence to: Jenny Murfield, Menzies Health Institute Queensland, Griffith University, Health Sciences (N48), 170 Kessels Road, Nathan, Brisbane, Queensland, 4111, Australia. Tel: +61 (0)7 37557855 Email: j.murfield@griffith.edu.au 


\section{The Role of Self-Compassion, Dispositional Mindfulness, and Emotion Regulation in the Psychological Health of Family Carers of Older Adults}

Objectives: This study explored the role of compassion and dispositional mindfulness in the psychological health of family carers of older adults and tested for potential mediating effects of emotion regulation difficulties and adaptive coping strategies. Methods: A sample of 141 family carers of adults aged 65 years or older with chronic conditions completed a cross-sectional survey between July - December 2019. The survey included self-report scales that measured: self-compassion, compassion for others, compassion from others, dispositional mindfulness, depression, anxiety, stress, emotion- and problem-focused coping strategies, and difficulties in emotion regulation. Results: Path analyses found that increased self-compassion and increased dispositional mindfulness was associated with lower psychological distress, and that this was mediated by reduced difficulties in emotion regulation. The model had excellent fit, explaining $64.8 \%$ of the variance in psychological distress, and $52.2 \%$ of the variance in emotion regulation difficulties. Conclusions: Self-compassion and dispositional mindfulness may help buffer the psychological distress of family carers of older adults, and adaptive emotion regulation is an important mechanism of change in these relationships. Clinical Implications: Interventions that aim to cultivate selfcompassion and mindfulness could be clinically useful in reducing psychological distress within populations of family carers of older adults by promoting adaptive emotion regulation.

Keywords: depression, anxiety, stress, caregiving, path analysis 


\section{Introduction}

It is well established that caring for an older family member can be a demanding and often stressful experience. Research shows that the occurrence of psychological distress is higher in family caregivers (i.e., family, friend, or neighbour) than non-caregivers (Collins \& Kishita, 2019; Stacey et al., 2018), and that poor carer mental health predicts increased suicidal ideation (Joling et al., 2018) and increased neglect and abuse of the care recipient (Kohn \& Verhoek-Oftedahl, 2011). These compelling data have heightened calls for family carers to be better supported in ways that more adequately meet their unmet needs. One key unmet need family carers have self-identified is greater access to professional support for emotional coping and stress management (Silva et al., 2013). For this reason, targeted psychological support programs have been increasingly studied to investigate their effectiveness in this population group and, most recently, the psychological processes of mindfulness and compassion have been explored.

Mindfulness and compassion are complex multidimensional constructs that are conceptualised and defined in various ways; however, there is some agreement on the principal characteristics of each. Broadly, mindfulness is defined as 'the awareness that emerges through paying attention on purpose, in the present moment, and nonjudgmentally to the unfolding of experience moment by moment' (Kabat-Zinn, 2003, p. 145). Compassion is often regarded as a dynamic three-way 'flow' involving compassion for self, compassion for others, and compassion from others. Within this understanding, compassion is defined as 'a sensitivity to suffering in self and others, with a commitment to try to prevent or alleviate it' (Gilbert, 2014, p.19).

A growing body of research has sought to explore the relationship between dispositional mindfulness/compassion and psychological health, and in doing so, an important and potentially protective role has been demonstrated. Across the lifespan, 
dispositional mindfulness and compassion have shown positive associations with wellbeing and negative associations with depression, anxiety, and stress (e.g., MacBeth \& Gumley, 2012; Tomlinson et al., 2018). Similar findings have emerged in the few studies conducted with family caregiver groups, with self-compassion negatively associated with burden (Lloyd et al., 2019), and self-compassion and dispositional mindfulness identified as predictors of depression (Hlabangana \& Hearn, 2019; Hsieh et al., 2019). Building on these promising findings, research has begun to focus on the specific mechanisms through which compassion and dispositional mindfulness operate, and early evidence suggests emotion regulation and adaptive coping strategies play an important role.

Emotion regulation refers to a range of automatic and deliberate cognitive, behavioural, and physiological processes that interact to affect how an individual experiences the expression, intensity, and duration of an emotion (Gratz \& Roemer, 2004). Although similar, coping strategies are more targeted in scope, referring to the various means by which an individual attempts to specifically manage a stressful experience, and these can be either helpful (i.e., reducing emotional distress, changing the situation) or unhelpful strategies (i.e., blame or denial) (Carver et al., 1989). Within the literature, both emotion regulation and ways of coping have been found to impact psychological health, with more maladaptive forms of coping and emotion regulation independently associated with poor mental health, and more adaptive forms appearing to buffer the effects of psychopathology (e.g., Aldao et al., 2010; Berking \& Whitley, 2014; Gratz \& Roemer, 2004). There is also growing evidence that emotion regulation and coping strategies may be important mechanisms of change in the compassion/mindfulness-psychological health relationship (e.g., Finkelstein-Fox et al., 2019; Gratz \& Tull, 2010; Inwood \& Ferrari, 2018; Neff et al., 2005). In family carers of people with dementia, dysfunctional coping strategies have been found to mediate the link between self-compassion and burden (Lloyd et al., 2019). However, this is the only known study 
conducted to date with a sample of family carers, and there is a need for more research to investigate whether compassion and dispositional mindfulness may facilitate adaptive emotion regulation and coping to buffer against the psychological impacts of caring.

The present study aims to explore the associations between compassion (to self, for others, from others), dispositional mindfulness, emotion regulation difficulties, use of adaptive coping strategies (emotion- and problem-focused), and psychological distress (depression, anxiety, and stress) within family carers of older adults. It also aims to test the possible mechanism of change in the links between compassion/dispositional mindfulness and psychological distress by specifically examining whether emotion regulation difficulties and use of adaptive coping strategies have a mediating role. We hypothesise the following: (1) Compassion and dispositional mindfulness will be negatively associated with emotion regulation difficulties and psychological distress, but positively associated with adaptive coping strategies; and (2) When tested in a structural equation model, emotion regulation difficulties and use of adaptive coping strategies will mediate the relationship between compassion/dispositional mindfulness and psychological distress.

\section{Method}

\section{Participants and Procedure}

A cross-sectional survey was available for completion by any adult (aged 18 years or older) who self-identified as an informal carer providing regular, ongoing, unpaid care to a family member, friend, or neighbour aged 65-years or older, and were able to read and write in English (Table 1). Although the survey was primarily promoted within Australia via community and volunteer organisations, face-to-face presentations, radio interviews, print newspaper advertisements, social media, and word-of-mouth, it is possible that some of these methods also recruited participants from any country around the world. Participants could 
complete the survey online (hosted through LimeSurvey GmbH, Hamburg, Germany), on paper (with pre-paid postal return), or verbally with the lead author.

Ethical approval was obtained from Griffith University Human Research Ethics Committee (GU ref. no. 2019/481). Participants were given written information about the survey's purpose and were informed that completion represented consent, that survey data were anonymous, that their involvement was voluntary, and that they could stop at any time. Participants were encouraged to take breaks during the survey, and there were reminders at two points to pause completion if needed. Telephone and online contacts of mental health support organisations around the world were also provided.

In June 2019, the survey was piloted in two rounds with seven family carers. After round one $(n=3)$, minor edits were made to the information text and two demographic questions. No changes were made after round two, and these data $(n=4)$ are included in the final sample. The survey was open from July - December 2019.

\section{Measures}

The survey included questions about the demographic characteristics of the family carer and the older adult care recipient, and seven self-report scales.

\section{Compassionate Engagement and Action Scales}

The Compassionate Engagement and Action Scales (CEAS) (Gilbert et al., 2017) are three 13-item scales that individually assess Compassion for Self, Compassion for Others, and Compassion from Others. Within all scales, items are rated for frequency of occurrence using a 10 -point Likert scale $(1=$ never to $10=$ always $)$. Three items in each scale are reverse statements and, as per the original scoring instructions, these are excluded from scoring. Scores for each scale are summated for an overall assessment of each orientation of 
compassion, and for two sub-scale assessments of compassionate engagement and compassionate actions. There is also an option within the Compassion for Self scale to assess compassionate engagement more specifically in terms of sensitivity to suffering and engagement with suffering. Higher scores indicate higher levels of compassion. The CEAS have performed acceptably within compassion-focused research and have demonstrated good-to-excellent levels of internal reliability (e.g., Gilbert et al., 2017; Hsieh et al., 2019; Steindl et al., 2018; Steindl et al., 2019). As part of a larger program of research, we have also confirmed the validity of the CEAS with family carers of older adults (Murfield et al., 2020). Within the current study, we used total scale scores for an assessment of each compassion orientation.

\section{Mindful Attention Awareness Scale}

The Mindful Attention Awareness Scale (MAAS) (Brown \& Ryan, 2003) consists of 15items that measure attention to and awareness of the present moment in everyday life. All items are statements representing the opposite of mindfulness and are rated for frequency of occurrence using a 6-point Likert scale $(1=$ almost always to $6=$ almost never $)$. A mean score is calculated for an overall assessment of dispositional mindfulness. Higher scores indicate greater levels of dispositional mindfulness. The MAAS has good psychometric properties and has been used widely within research (Park et al., 2013), including in studies involving family carers of people living with dementia (e.g., Bormann et al., 2009; Danucalov et al., 2017).

\section{Depression, Anxiety, and Stress Scales - 21}

The Depression Anxiety Stress Scales - 21 (DASS-21) (Lovibond \& Lovibond, 1995), which is a shorter version of the original DASS, consists of 21 -items that measure, in three 
sub-scales, the emotional states of depression, anxiety, and stress. Items are rated for frequency of occurrence over the previous one-week period using a 4-point Likert scale $(0=$ did not apply to me at all to $3=$ applied to me very much or most of the time). Scores for each subscale are summated and multiplied by two (to enable comparison with the full 42-item DASS) to produce an assessment of each construct. Higher scores represent greater severity of each emotional state, and manualised cut-off scores are available for clinical classification into normal, mild, moderate, severe, and extremely severe categories. The DASS-21 has been used extensively in research with clinical and non-clinical population, including family carers of older adults with various chronic conditions (e.g., Ervin et al., 2015; O'Connell et al., 2010).

\section{Brief Coping Orientations to Problems Experienced Scale}

The Brief Coping Orientations to Problems Experienced Scale (COPE) (Carver, 1997) consists of 28 -items that measure the use of different coping strategies at times of stress. Items are rated for frequency of use via a 4-point Likert scale (1 = I haven't been doing this at all to $4=I$ 've been doing this a lot). Scores can be summated as a measure of 14 different coping strategies, and these can be combined to provide an assessment of three broader coping strategy categories: emotion-focused (use of emotional support, positive reframing, humour, acceptance, religion); problem-focused (active coping, use of instrumental support, planning); and dysfunctional coping (self-distraction, denial, substance use, behavioural disengagement, venting, self-blame) (Coolidge et al., 2000). In all subscales, higher scores reflect greater use of the coping strategy. The scale has been used extensively within caregiver research, and has been used in studies looking at compassion fatigue and selfcompassion in family carers of older adults living with dementia and chronic conditions (e.g., 
Lloyd et al., 2019; Lynch et al., 2018). Within the current study, we focused on adaptive coping and used the emotion- and problem-focused coping subscales only.

\section{Difficulties in Emotion Regulation Scale}

The Difficulties in Emotion Regulation Scale (DERS) (Gratz \& Roemer, 2004) consists of 36-items that assess difficulties in emotion regulation. Items are rated for frequency of occurrence using a 5-point Likert scale $(1=$ never to $5=$ almost always $)$. Scores are summated (with reverse scoring applied to 11 items) to produce an overall assessment of emotion regulation difficulties, and for six subscale assessments of: non-acceptance of emotional responses; difficulties engaging in goal-directed behaviour; impulse control difficulties; lack of emotional awareness; limited access to emotion regulation strategies; and lack of emotional clarity. Higher scores represent greater difficulty with emotion regulation. The DERS has been used extensively within research with diverse clinical and non-clinical populations, including studies involving family caregivers of adults living with dementia and chronic illness (e.g., O’Toole et al., 2019; Romero-Moreno et al., 2017). Within the current study, we used the total score to assess emotion regulation difficulties.

\section{Data Analyses}

The survey was completed by 259 participants (239 online, 20 paper with pre-paid postal return, 0 verbally). Online responses were automatically converted for analysis in IBM SPSS Version 26 (IBM Corp, Armonk, NY), and hard copy data were manually entered. Frequency statistics were inspected for missing values, distribution spread, and outliers. Upon examination, 118 cases were removed: 62 contained demographic data only, 45 had more than $20 \%$ missing data, and 11 did not meet participant inclusion criteria. In the final sample of 141 , which equated to a completion rate of $54.5 \%$, missing data were minimal $(<1 \%$ 
overall and within each scale); appeared to be Missing Completely at Random (Little's MCAR test $p>.05$ overall and for each scale); and was approximated with a normal distribution (no absolute skew value > 2.0 and no absolute kurtosis value > 7.0 (Curran et al., 1996)). The expectation-maximization algorithm was used to impute missing data for each scale; no imputation was applied to demographic variables.

To explore associations between all study variables, bivariate correlational analyses were undertaken. To assess the potential confounding effects of demographic variables on carers' depression, anxiety, and stress, we ran separate multivariable regression models (creating dummy coding for variables with more than two response categories). Significant explanatory variables were identified using the enter method first and repeated using the stepwise method. Age of the family carer was the only statistically significant variable examined, and this was included as a covariable in the subsequent structural equation modelling. Effect size $\left(R^{2}\right)$ was interpreted using conventional definitions set out by Cohen (1992).

To test the relationship between study variables and hypothesised mediator effects, a path analysis was undertaken in IBM SPSS AMOS Version 26.0 (IBM Corp, Armonk, NY). As structural equation modelling is conducted on complete-case data, two participants with missing data for demographic variables were excluded and the path analysis was conducted on 139 complete participant cases. Using maximum likelihood estimation, the initial model included five observed exogenous variables (self-compassion; compassion for others; compassion from others; dispositional mindfulness; age of family carer), six observed endogenous variables (emotion regulation difficulties; emotion- and problem-focused coping; depression; anxiety; stress), and one unobserved endogenous variable (psychological distress). Model goodness-of-fit was tested using the following indices: chi-square statistic $\left(\chi^{2}\right)$ with non-significant values of $p>.05$; Normed Chi-Square statistic $\left(\chi^{2} / \mathrm{df}\right)$ values of $<3$; 
Root Mean Squared Error of Approximation (RMSEA) value of < .06; Standardized Root Mean Square Residual (SRMR) values of < .08; Comparative Fit Index (CFI), Tucker Lewis Index (TLI) and Goodness-of-Fit Index (GFI) values of $\geq .95$ (Byrne, 2010; Hu \& Bentler, 1999; Wheaton et al., 1977). All statistically non-significant pathways were removed, and the model re-run. Bootstrapping resampling with 5,000 samples and bias-corrected $95 \%$ confidence intervals were computed for total, direct, and indirect effects. To test the adequacy of our sample size for the final model produced we used the Hoelter Index. This test yielded a value of $245(p=.01)$, which exceeded the conventional adequacy criteria of 200, and thereby confirmed that our sample size was adequate for the final model produced.

\section{Results}

A total of 141 family carers from six countries completed the survey (Table 1). Participants were typically female, had an average age of 60 years $(S D=11.5)$, resided in Australia, had post-secondary qualifications, and were either retired or in employment. The majority identified as the primary carer of a female parent or partner who had an average age of 81 years $(S D=9.0)$, and lived with dementia, a musculoskeletal disorder, and/or cardiovascular disease. Most participants lived full-time with the older adult and had been in the caring role for an average of 6 years $(S D=5.5)$.

\section{[Insert Table 1]}

The descriptive statistics and internal consistencies of all measures used in the study are shown in Table 2. Notably, when looking at the psychological health of family carers in our sample, mean scores for depression, anxiety, and stress all met the threshold for moderate severity of symptoms. Moreover, as shown in Table 3, the proportion of participants meeting 
the thresholds for severe/extremely severe symptoms was considerable: $53.2 \%$ for anxiety, $34 \%$ for depression, and $28.4 \%$ for stress.

\section{[Insert Tables $2 \& 3$ ]}

\section{Correlational Analyses}

Correlations between all study variables are presented in Table 4. In terms of psychological health, there were significant associations with depression, anxiety, and stress and all study variables except two (emotion- and problem-focused coping with anxiety). The strongest positive associations were between depression, anxiety, and stress themselves (from $r=.62$ to $r=.75, p<.01$ ). Difficulties in emotion regulation had the next strongest and positive association with all three negative emotional states (from $r=.59$ to $r=.70, p<.01$ ), followed by dispositional mindfulness (negative associations from $r=-.48$ to $r=-.58, p<.01$ ) and self-compassion (negative associations from $r=-.34$ to $r=-.46, p<.01$ ). Specifically, dispositional mindfulness had moderate negative correlations with depression $(r=-.48)$, anxiety $(r=-.50)$, and stress $(r=-.58)$, whereas self-compassion had a moderate negative correlation with depression $(r=-.46)$, but weaker negative correlations with anxiety $(r=-.34)$ and stress $(r=-.38)$. The remaining variables had limited associations with psychological health.

When considering how emotion regulation difficulties and coping strategies related to dispositional mindfulness and the three orientations of compassion, the strongest negative associations were between emotion regulation difficulties and both self-compassion $(r=-$ $.59, p<.01)$ and dispositional mindfulness $(r=-.58, p<.01)$. Emotion-and problem-focused coping had a moderate-to-weak positive association with self-compassion $(r=.38 \& r=.46$, $p<.01$ ), and only problem-focused coping had a weak positive association with dispositional mindfulness $(r=.21, p<.05)$. Compassion from others had a moderate-to-weak positive 
correlation with both problem- and emotion-focused coping $(r=.46 \& r=.36, p<.01)$, and a weak negative association with emotion regulation difficulties $(r=-.30, p<.01)$. Conversely, compassion for others had no association with coping strategies or emotion regulation difficulties (all $p>.05$ ).

For the three orientations of compassion, there were significant moderate-to-weak positive correlations between self-compassion and compassion for others $(r=.19, p<.05)$ and compassion from others $(r=.44, p<.01)$. However, no significant correlation was found between giving compassion to others and receiving compassion from others $(p>0.05)$. There was also evidence of different relationships between dispositional mindfulness and each compassion orientation: self-compassion had a weak positive association with dispositional mindfulness $(r=.36, p<.01)$; compassion for others had a weak negative correlation $(r=-.21, p<.05)$; and compassion from others had no association $(p>.05)$.

[Insert Table 4]

\section{Covariables}

The potential influence of demographic variables on carers' depression, anxiety, and stress was explored through multiple regression analyses that included all variables listed in Table 1 as potentially explanatory variables. Age of family carer was the only significant predictor of psychological health; however, its effect size was small, accounting for $5.7 \%$ of the variance in depression $(F(1,124)=7.52, p=.007), 9.8 \%$ in anxiety $(F(1,124)=13.41, p<.001)$, and $5.2 \%$ in stress $(F(1,124)=6.80, p=.010)$. Being older was significantly associated with fewer symptoms of depression, anxiety, and stress (i.e., for every year increase in age there is a: $\beta=-0.24$ unit decrease in depression; $\beta=-0.31$ unit decrease in anxiety; and $\beta=-0.23$ decrease in stress), hence we included family carer age as a covariable in the structural equation modelling. 


\section{Path Analysis}

The possible mediating effects of difficulties in emotion regulation and adaptive coping strategies on the relationship between psychological distress and the three orientations of compassion and dispositional mindfulness (when controlling for age of the carer) were explored in a path analysis. In the initial model, pathways for compassion for others, compassion from others, and emotion- and problem-focused coping were not statistically significant. These variables were removed from the analysis and the model was readjusted. After further testing for significant partial and full mediation pathways, the final adjusted model was confirmed, and all pathways were statistically significant $(p<.01)$ (Figure $1 \&$ Supplementary data). This model had excellent fit with the sample data $\left(\chi^{2} \mathrm{p}=.22 ; \chi^{2} / \mathrm{df}=\right.$ $1.31 ; \mathrm{RMSEA}=.05 ; \mathrm{SRMR}=.03 ; \mathrm{CFI}=.99 ; \mathrm{TLI}=.99 ;$ and $\mathrm{GFI}=.97)$, and explained $64.8 \%$ of the variance in psychological distress, and $52.2 \%$ of the variance in difficulties in emotion regulation. In terms of our second hypothesis, difficulties in emotion regulation fully mediated the relationship between self-compassion and psychological distress, but partially mediated the relationship between dispositional mindfulness and psychological distress. Specifically, increased self-compassion $(\beta=-.26, p<.001,95 \% \mathrm{CI}=-.35$ to -.18$)$, increased dispositional mindfulness $(\beta=-.25, p<.001,95 \% \mathrm{CI}=-.35$ to -.16$)$, and increased age of the $\operatorname{carer}(\beta=-.11, p=.004,95 \% \mathrm{CI}=-.18$ to -.03$)$ were associated with lower psychological distress, and this was mediated by reduced difficulties in emotion regulation. However, only increased dispositional mindfulness was also associated with lower psychological distress directly $(\beta=-.26, p=.003,95 \% \mathrm{CI}=-.41$ to -.11$)$.

\section{[Insert Figure 1]}




\section{Discussion}

This study sought to explore the role of compassion and dispositional mindfulness in the psychological health of family carers of older adults and to test for the potential mediating effects of emotion regulation difficulties and adaptive coping strategies. We found that selfcompassion and dispositional mindfulness may help reduce the psychological distress of family carers of older adults, and that adaptive emotion regulation is an important mechanism of change in the relationships. These findings provide preliminary evidence of the applicability of targeting self-compassion and mindfulness in interventions to support family carers' psychological health, and this has implications for both research and practice.

A growing number of studies conducted with various populations (e.g., Finlay-Jones et al., 2015; Gilbert et al., 2017; Prakash et al., 2015; Steindl et al., 2018), including groups of family carers (Hlabangana \& Hearn, 2019; Hsieh et al., 2019; Lloyd et al., 2019), have documented important links between aspects of compassion, dispositional mindfulness, emotion regulation, coping strategies, and psychological health. Within our study, we found evidence of similar relationships, and these were largely in line with our first hypothesis. We found that self-compassion, compassion from others, and dispositional mindfulness were inversely associated with emotion regulation difficulties and psychological distress, and positively associated with adaptive coping strategies. Of these relationships, psychological distress had the strongest positive association with emotion regulation difficulties, followed by negative associations with dispositional mindfulness and self-compassion. However, we also found that compassion for others was not correlated with the variables in the same way: it had no significant association with either of the coping strategies or emotion regulation difficulties, but it had significant positive associations with depression, anxiety, and stress. These correlations were very weak and, consistent with previous research (Gilbert et al., 2017; Steindl et al., 2018), there was no evidence that compassion for others was related to 
psychological distress when tested in a structural equation model. Nevertheless, the presence of some relationship (in a positive direction) is notable and, for our study population, interpretation may be understood using the suffering-compassion model of caregiver stress (Schulz et al., 2007). In this model, when the caregiver is unable to reduce the suffering of the care recipient, greater levels of compassion (to the care recipient) leads to more psychological distress. Within our study, it is possible that the question of compassion for others was answered in relation to the care recipient. As participants were caring for a family member with a chronic illness, this may explain why we saw higher levels of compassion for others reported and, ultimately, why there was evidence of some relationship between greater compassion for others and greater psychological distress.

Emotion regulation has been identified as the potential mechanism of change through which self-compassion and dispositional mindfulness may impact psychological distress (Guendelman et al., 2017; Inwood \& Ferrari, 2018) and, within family caregiver groups, dysfunctional coping has been shown to mediate the link between self-compassion and caregiver burden (Lloyd et al., 2019). However, work in this area is limited in number and scope, and we are unaware of any study that has explored the role of both emotional regulation and coping strategies within the context of the three orientations of compassion, or when looking at compassion and dispositional mindfulness together. To this end, we tested a structural equation model exploring the mediating role of emotion regulation difficulties and emotion- and problem-focused coping strategies in the relationship between compassion/dispositional mindfulness and psychological distress. After controlling for age of the family carer, we found that only self-compassion and dispositional mindfulness were associated with psychological distress, and that difficulties in emotion regulation (but not adaptive coping strategies) mediated these relationships. Difficulties in emotion regulation had a full mediating effect for self-compassion and was therefore the mechanism of change. 
However, dispositional mindfulness had a direct impact on psychological distress, meaning difficulties in emotion regulation had only a partial mediating effect. These results support previous research that suggests the compassion/dispositional mindfulness-psychological distress relationship can be understood through an emotion regulation framework (Guendelman et al., 2017; Inwood \& Ferrari, 2018). It also reinforces current understanding that, although self-compassion and dispositional mindfulness impact psychological health through similar pathways, they are distinct constructs that warrant individual assessment (Germer \& Barnhofer, 2017; Neff \& Dahm, 2015). In terms of the absence of a mediating effect of adaptive coping strategies, our results are similar to those reported in a study involving family carers of people living with dementia (Lloyd et al., 2019). When taken together, this suggests that emotion regulation, rather than adaptive coping strategies, is the primary mechanism of change through which self-compassion and dispositional mindfulness impact psychological health.

Another important result arising from our modelling is that compassion for others and compassion from others had no significant association with psychological distress. Although this is a similar result to earlier research (Gilbert et al., 2017; Hermanto \& Zuroff, 2018; Steindl et al., 2018), it is also somewhat unexpected, as evidence shows that being compassionate to others and also receiving compassion from others can afford some psychological health benefits (Hermanto et al., 2016; Mongrain et al., 2011). In understanding the absence of a relationship between compassion for others with psychological distress, as previously discussed, the suffering-compassion model of caregiver distress may offer some explanation (e.g., carers may have answered the questions about compassion to others in relation to the caregiving content specifically, whereby suffering and compassion have distinct roles (Schulz et al., 2007)). Regarding the non-association of compassion from others, we draw on previous explanations that suggest findings may be due, 
in part, to how this aspect is measured by the CEAS (Gilbert et al., 2017; Steindl et al., 2018). Specifically, rather than assessing the individual's openness to receiving compassion from others - which when assessed as fears of compassion (e.g., receiving compassion is indulgent, a weakness, will be judged for it) is related to depression, shame, and self-criticism (Kirby et al., 2019) - the CEAS may be assessing the availability of caregivers' significant others to be compassionate. As such, we recommend that fears of compassion be assessed in future work to explore this more specifically (e.g., through use of the Fears of Compassion Scales (Gilbert et al., 2011) to assess fears within each compassion orientation).

Finally, our study showed the complex relationship between the three orientations of compassion with each other and with dispositional mindfulness. Comparable to earlier work, we found that scores in one compassion orientation did not necessarily relate to scores on the other orientations (Gilbert et al., 2017), and we also found that dispositional mindfulness had a very different relationship with each compassion orientation. This reinforces the importance of measuring all aspects of compassion and dispositional mindfulness in cross-sectional work, and in assessing their impacts on psychological health individually.

\section{Limitations}

To the best of our knowledge, this is the first study to specifically explore the role of compassion and dispositional mindfulness in the psychological health of family carers of older adults, while also testing for potential mediating effects of emotion regulation difficulties and adaptive coping strategies. However, there are a number of important limitations that should be kept in mind when interpreting the results. First, despite the confirmed adequacy of our sample size for the final model, it was relatively small for structural equation modelling. Second, the cross-sectional nature of the study prevents causal inferences to be made about the relationship between variables. Third, as we used only self- 
report measures, uncontrolled subjective reporting bias may be present within our findings, and this may be particularly the case for reported levels of psychological distress. Fourth, survey participants were typically Australian women who provided informal primary care to a female parent or partner with dementia. It's possible that this profile of participants may be due to our recruitment strategy and the organisations we targeted to help with promotional activities. As such, generalisability of the results beyond this group are limited.

\section{Conclusions}

This study found support for the potential role of self-compassion and dispositional mindfulness in the psychological health of family carers of older adults. It also showed that difficulties in emotion regulation is an important mechanism of change in these relationships. These findings lend support for the applicability of psychological interventions that aim to cultivate self-compassion and mindfulness as a means of promoting adaptive emotion regulation for psychological health within family carers of older adults. Further research with broader and larger samples is needed to replicate and build on this initial evidence.

Additionally, more research is required to evaluate current interventions which specifically target self-compassion and dispositional mindfulness with this population (e.g., Mindful SelfCompassion (Neff \& Germer, 2013), Compassion-Focused Therapy (Gilbert, 2010); and Compassionate Mind Training (Irons \& Beaumont, 2017)). Current evidence about effective use of compassion- and mindfulness-based interventions with the family carer population is limited (Murfield et al., 2019), and we advocate that more rigorous evaluations are necessary to advance understanding in this emerging area.

More broadly, our findings also underline the considerable emotional distress that many family carers experience. On average, participants in our study reported symptoms of depression, anxiety, and stress at moderate severity, and there was a considerable proportion 
that met the thresholds for severe/extremely severe symptoms. Such data adds to the extant literature and reinforces, once again, the importance of providing psychological support to this population.

\section{Clinical Implications}

- Interventions that aim to cultivate self-compassion and mindfulness could be clinically useful in reducing psychological distress within populations of family carers of older adults by promoting adaptive emotion regulation.

- Assessing levels of self-compassion and dispositional mindfulness could be a helpful way of identifying family carers who are at risk of experiencing psychological distress and emotion regulation difficulties.

\section{Acknowledgements}

The authors thank the family carers who generously took the time to complete the survey, and the organisations, services, and online support groups who helped with promotional activities.

\section{Declarations of Interest Statement}

The authors declare that they have no conflict of interest.

\section{Funding Information}

This work was supported by a Griffith University Postgraduate Scholarship. 


\section{Author Contributions}

JM conceived and designed the study, undertook data collection and statistical analysis, and wrote, revised, and finalised the paper. WM and AOD supervised the study, providing advice in aspects of study design and data collection, and conceptually commented on and revised the paper. RW provided statistical advice on data analysis, and conceptually commented on and revised the paper. All authors read and approved the final manuscript. 


\section{References}

Aldao, A., Nolen-Hoeksema, S., \& Schweizer, S. (2010). Emotion-regulation strategies across psychopathology: A meta-analytic review. Clinical Psychology Review, 30(2), 217-237. https://doi.org/10.1016/j.cpr.2009.11.004

Berking, M., \& Whitley, B. (2014). Affect regulation training: A practitioner's manual. Springer. https://doi.org/10.1007/978-1-4939-1022-9

Bormann, J., Warren, K. A., Regalbuto, L., Glaser, D., Kelly, A. N. N., Schnack, J., \& Hinton, L. (2009). A spiritually based caregiver intervention with telephone delivery for family caregivers of veterans with dementia. Family and Community Health, 32(4), 345-353. https://doi.org/10.1097/FCH.0b013e3181b91fd6.

Brown, K. W., \& Ryan, R. M. (2003). The benefits of being present: Mindfulness and its role in psychological well-being. Journal of Personality and Social Psychology, 84(4), 822-848. https://doi.org/10.1037/0022-3514.84.4.822

Byrne, B. (2010). Structural equation modeling with AMOS: Basic concepts, applications, and programming (2nd ed.). Routledge.

Carver, C. S. (1997). You want to measure coping but your protocol's too long: Consider the brief COPE. International Journal of Behavioral Medicine, 4(1), 92-100. https://doi.org/10.1207/s15327558ijbm0401_6

Carver, C. S., Scheier, M. F., \& Weintraub, J. K. (1989). Assessing coping strategies: A theoretically based approach. Journal of Personality and Social Psychology, 56(2), 267-283. https://doi.org/10.1037//0022-3514.56.2.267

Cohen, J. (1992). A power primer. Psychological Bulletin, 112(1), 155-159. https://doi.org/10.1037//0033-2909.112.1.155 
Collins, R. N., \& Kishita, N. (2019). Prevalence of depression and burden among informal care-givers of people with dementia: A meta-analysis. Ageing and Society, 1-38. https://doi.org/10.1017/S0144686X19000527

Coolidge, F. L., Segal, D. L., Hook, J. N., \& Stewart, S. (2000). Personality disorders and coping among anxious older adults. Journal of Anxiety Disorders, 14(2), 157-172. https://doi.org/10.1016/s0887-6185(99)00046-8

Curran, P. J., West, S. G., \& Finch, J. F. (1996). The robustness of test statistics to nonnormality and specification error in confirmatory factor analysis. Psychological Methods, 1(1), 16-29. https://doi.org/10.1037/1082-989X.1.1.16

Danucalov, M. A. D., Kozasa, E. H., Afonso, R. F., Galduroz, J. C. F., \& Leite, J. R. (2017). Yoga and compassion meditation program improve quality of life and selfcompassion in family caregivers of Alzheimer's disease patients: A randomized controlled trial. Geriatrics \& Gerontology International, 17(1), 85-91. https://doi.org/10.1111/ggi.12675

Ervin, K., Pallant, J., \& Reid, C. (2015). Caregiver distress in dementia in rural Victoria. Australasian Journal on Ageing, 34(4), 235-240. https://doi.org/10.1111/ajag.12158

Finkelstein-Fox, L., Park, C. L., \& Riley, K. E. (2019). Mindfulness' effects on stress, coping, and mood: A daily diary goodness-of-fit study. Emotion, 19(6), 1002-1013. https://doi.org/10.1037/emo0000495

Finlay-Jones, A. L., Rees, C. S., \& Kane, R. T. (2015). Self-compassion, emotion regulation and stress among Australian psychologists: Testing an emotion regulation model of self-compassion using structural equation modeling. PloS One, 10(7), e0133481. https://doi.org/10.1371/journal.pone.0133481 
Germer, C., \& Barnhofer, T. (2017). Mindfulness and compassion: Similaritlies and differences. In P. Gilbert (Ed.), Compassion: Concepts, research and applications (pp. 69-86). Routledge.

Gilbert, P. (2010). Compassion focused therapy: Distinctive features. Taylor and Francis Ltd.

Gilbert, P., Catarino, F., Duarte, C., Matos, M., Kolts, R., Stubbs, J., Ceresatto, L., Duarte, J., Pinto-Gouveia, J., \& Basran, J. (2017). The development of compassionate engagement and action scales for self and others. Journal of Compassionate Health Care, $4(1), 4$.

Gilbert, P., McEwan, K., Matos, M., \& Rivis, A. (2011). Fears of compassion: Development of three self-report measures. Psychology and Psychotherapy: Theory, Research and Practice, 84(3), 239-255. https://doi.org/10.1348/147608310X526511

Gratz, K. L., \& Roemer, L. (2004). Multidimensional assessment of emotion regulation and dysregulation: Development, factor structure, and initial validation of the difficulties in emotion regulation scale. Journal of Psychopathology and Behavioral Assessment, $26(1), 41-54$.

Gratz, K. L., \& Tull, M. T. (2010). Emotion regulation as a mechanism of change in acceptance- and mindfulness-based treatments. In R. A. Baer (Ed.), Assessing mindfulness and acceptance processes in clients: Illuminating the theory and practice of change. (pp. 107-133). New Harbinger Publications.

Guendelman, S., Medeiros, S., \& Rampes, H. (2017). Mindfulness and emotion regulation: Insights from neurobiological, psychological, and clinical studies. Frontiers in Psychology, 8, 220. https://doi.org/10.3389/fpsyg.2017.00220

Hermanto, N., \& Zuroff, D. C. (2018). Experimentally enhancing self-compassion: Moderating effects of trait care-seeking and perceived stress. The Journal of Positive Psychology, 13(6), 617-626. https://doi.org/10.1080/17439760.2017.1365162 
Hermanto, N., Zuroff, D. C., Kopala-Sibley, D. C., Kelly, A. C., Matos, M., Gilbert, P., \& Koestner, R. (2016). Ability to receive compassion from others buffers the depressogenic effect of self-criticism: A cross-cultural multi-study analysis. Personality and Individual Differences, 98, 324-332. https://doi.org/10.1016/j.paid.2016.04.055

Hlabangana, V., \& Hearn, J. H. (2019). Depression in partner caregivers of people with neurological conditions; associations with self-compassion and quality of life. Journal of Mental Health, 29(2), 176-181. https://doi.org/10.1080/09638237.2019.1630724

Hsieh, C.-C., Yu, C.-J., Chen, H.-J., Chen, Y.-W., Chang, N.-T., \& Hsiao, F.-H. (2019). Dispositional mindfulness, self-compassion, and compassion from others as moderators between stress and depression in caregivers of patients with lung cancer. Psycho-Oncology, 28(7), 1498-1505. https://doi.org/10.1002/pon.5106

Hu, L., \& Bentler, P. M. (1999). Cutoff criteria for fit indexes in covariance structure analysis: Conventional criteria versus new alternatives. Structural equation modeling: A multidisciplinary journal, 6(1), 1-55. https://doi.org/10.1080/10705519909540118

Inwood, E., \& Ferrari, M. (2018). Mechanisms of change in the relationship between selfcompassion, emotion regulation, and mental health: A systematic review. Applied Psychology: Health and Well-Being, 10(2), 215-235.

https://doi.org/10.1111/aphw.12127

Irons, C., \& Beaumont, E. (2017). The compassionate mind workbook: A step-by-step guide to developing your compassionate self. Little Brown Book Group.

Joling, K. J., O'Dwyer, S. T., Hertogh, C. M. P. M., \& van Hout, H. P. J. (2018). The occurrence and persistence of thoughts of suicide, self-harm and death in family caregivers of people with dementia: A longitudinal data analysis over 2 years. 
International journal of geriatric psychiatry, 33(2), 263-270. https://doi.org/10.1002/gps.4708

Kabat-Zinn, J. (2003). Mindfulness-based interventions in context: Past, present, and future. Clinical Psychology: Science and Practice, 10(2), 144-156. https://doi.org/10.1093/clipsy.bpg016

Kirby, J. N., Day, J., \& Sagar, V. (2019). The 'flow' of compassion: A meta-analysis of the fears of compassion scales and psychological functioning. Clinical Psychology Review, 70, 26-39. https://doi.org/10.1016/j.cpr.2019.03.001

Kohn, R., \& Verhoek-Oftedahl, W. (2011). Caregiving and elder abuse. Medicine and Health, Rhode Island, 94(2), 47-49.

Lloyd, J., Muers, J., Patterson, T. G., \& Marczak, M. (2019). Self-compassion, coping strategies, and caregiver burden in caregivers of people with dementia. Clinical Gerontologist, 42(1), 47-59. https://doi.org/10.1080/07317115.2018.1461162

Lovibond, S. H., \& Lovibond, P. F. (1995). Manual for the Depression Anxiety \& Stress Scales (2nd ed.). Psychology Foundation.

Lynch, S. H., Shuster, G., \& Lobo, M. L. (2018). The family caregiver experience examining the positive and negative aspects of compassion satisfaction and compassion fatigue as caregiving outcomes. Aging \& Mental Health, 22(11), 14241431. https://doi.org/10.1080/13607863.2017.1364344

MacBeth, A., \& Gumley, A. (2012). Exploring compassion: A meta-analysis of the association between self-compassion and psychopathology. Clinical Psychology Review, 32(6), 545-552. https://doi.org/10.1016/j.cpr.2012.06.003

Mongrain, M., Chin, J. M., \& Shapira, L. B. (2011). Practicing compassion increases happiness and self-esteem. Journal of Happiness Studies, 12(6), 963-981. https://doi.org/10.1007/s10902-010-9239-1 
Murfield, J., Moyle, W., \& O'Donovan, A. (2019). Mindfulness- and compassion-based interventions for family carers of older adults: A scoping review. International Journal of Nursing Studies, 103495. https://doi.org/10.1016/j.ijnurstu.2019.103495

Murfield, J., Moyle, W., O'Donovan, A., \& Ware, R. S. (2020). Validity of the Compassionate Engagement and Action scales with family carers of older adults: Confirmatory factor analyses. International Psychogeriatrics, 1-11. https://doi.org/10.1017/S1041610220001738

Neff, K. D., \& Dahm, K. A. (2015). Self-compassion: What it is, what it does, and how it relates to mindfulness. In B. D. Ostafin, M. D. Robinson, \& B. P. Meier (Eds.), Handbook of mindfulness and self-regulation (pp. 121-137). Springer.

Neff, K. D., \& Germer, C. K. (2013). A pilot study and randomized controlled trial of the mindful self-compassion program. Journal of Clinical Psychology 69(1), 28-44. https://doi.org/10.1002/jclp.21923

Neff, K. D., Hsieh, Y.-P., \& Dejitterat, K. (2005). Self-compassion, achievement goals, and coping with academic failure. Self and identity, 4(3), 263-287. https://doi.org/10.1080/13576500444000317

O'Connell, B., Heslop, L., \& Fennessy, H. (2010). An evaluation of a wellness guide for older carers living in the community. Public Health Nursing, 27(4), 302-309. https://doi.org/10.1111/j.1525-1446.2010.00859.x

O’Toole, M. S., Mennin, D. S., Applebaum, A., Weber, B., Rose, H., Fresco, D. M., \& Zachariae, R. (2019). A randomized controlled trial of emotion regulation therapy for psychologically distressed caregivers of cancer patients. JNCI Cancer Spectrum, 4(1), pkz074. https://doi.org/10.1093/jncics/pkz074 
Park, T., Reilly-Spong, M., \& Gross, C. R. (2013). Mindfulness: A systematic review of instruments to measure an emergent patient-reported outcome (PRO). Quality of Life Research, 22(10), 2639-2659. https://doi.org/10.1007/s11136-013-0395-8

Prakash, R. S., Hussain, M. A., \& Schirda, B. (2015). The role of emotion regulation and cognitive control in the association between mindfulness disposition and stress. Psychology and Aging, 30(1), 160-171. https://doi.org/10.1037/a0038544

Romero-Moreno, R., Gallego-Alberto, L., Márquez-González, M., \& Losada, A. (2017). Psychometric properties of the Valued Living Questionnaire Adapted to Dementia Caregiving. Aging \& Mental Health, 21(9), 983-990. https://doi.org/10.1080/13607863.2016.1191055

Schulz, R., Hebert, R. S., Dew, M. A., Brown, S. L., Scheier, M. F., Beach, S. R., Czaja, S. J., Martire, L. M., Coon, D., Langa, K. M., Gitlin, L. N., Stevens, A. B., \& Nichols, L. (2007). Patient suffering and caregiver compassion: New opportunities for research, practice, and policy. Gerontologist, 47(1), 4-13. https://doi.org/10.1093/geront/47.1.4

Silva, A. L., Teixeira, H. J., Teixeira, M. J., \& Freitas, S. (2013). The needs of informal caregivers of elderly people living at home: An integrative review. Scandinavian Journal of Caring Sciences, 27(4), 792-803. https://doi.org/10.1111/scs.12019

Stacey, A. F., Gill, T. K., Price, K., \& Taylor, A. W. (2018). Differences in risk factors and chronic conditions between informal (family) carers and non-carers using a population-based cross-sectional survey in South Australia. BMJ Open, 8(7), e020173. https://doi.org/10.1136/bmjopen-2017-020173

Steindl, S. R., Matos, M., \& Creed, A. K. (2018). Early shame and safeness memories, and later depressive symptoms and safe affect: The mediating role of self-compassion. Current Psychology. https://doi.org/10.1007/s12144-018-9990-8 
Steindl, S. R., Yiu, R. X. Q., Baumann, T., \& Matos, M. (2019). Comparing compassion across cultures: Similarities and differences among Australians and Singaporeans. Australian Psychologist. https://doi.org/10.1111/ap.12433

Tomlinson, E. R., Yousaf, O., Vittersø, A. D., \& Jones, L. (2018). Dispositional mindfulness and psychological health: A systematic review. Mindfulness, 9(1), 23-43. https://doi.org/10.1007/s12671-017-0762-6

Wheaton, B., Muthen, B., Alwin, D. F., \& Summers, G. F. (1977). Assessing reliability and stability in panel models. Sociological Methodology, 8, 84-136.

https://doi.org/10.2307/270754 
Table 1. Participant demographic and caregiving characteristics

Characteristic Descriptive statistic

Age (years) ${ }^{\mathrm{a}}$ $59.6(11.5)$

Identifying gender ${ }^{\mathrm{b}}$

Female: Male

$121(85.8) ; 19(13.5)$

Highest level of education ${ }^{\mathrm{b}}$

University degree or higher

$63(44.7)$

Post-secondary certificate or diploma

$58(41.1)$

Secondary school

$19(13.5)$

Primary school

$1(0.7)$

Employment status ${ }^{\mathrm{b}}$

Employed

$49(34.8)$

Unemployed, not seeking work

26 (18.4)

Unemployed, seeking work

$6(4.3)$

Retired

$58(41.1)$

Student

$5(3.5)$

Country of residence ${ }^{b}$

Australia

107 (75.9)

United States of America

18 (12.8)

United Kingdom

$12(8.5)$

Canada

$1(0.7)$

China

$1(0.7)$

Sweden

$1(0.7)$

Relationship to older adult ${ }^{\mathrm{b}}$

Offspring

77 (54.6) 
Partner

Relative

Sibling

Friend or neighbour

Type of carer ${ }^{b}$

Primary: Secondary

Living full-time with older adult $\mathrm{t}^{\mathrm{b}}$

Yes: No

Length of time caring for older adult (years) ${ }^{\mathrm{a}}$

Age of older adult (years) ${ }^{\mathrm{a}}$

Identifying gender of older adult $\mathrm{t}^{\mathrm{b}}$

Female: Male

82 (58.2): 53 (37.6)

Main chronic conditions of older adult ${ }^{\mathrm{b}}$
Alzheimer's disease or dementia
$89(63.1)$
Musculoskeletal disorders
$46(32.6)$
Cardiovascular disease
$27(19.1)$
Cancer
$13(9.2)$
Parkinson's disease

Note. Frequencies and proportions may not add up to the total or $100 \%$ due to missing data, rounding, or multiple response options ${ }^{\mathrm{a}}$ continuous variables are reported as $M(S D)$

bategorical variables are reported as $n(\%)$

$n=141$ 
Table 2. Descriptive statistics and internal consistencies of study variables

\begin{tabular}{|c|c|c|}
\hline Variable (possible score) & $M(S D) ;$ Range & Cronbach's $\alpha$ \\
\hline CEAS: Compassion for Self $(10-100)$ & 60.7 (15.6); 20-95 & .85 \\
\hline CEAS: Compassion for Others (10-100) & 78.1 (13.6); 29-100 & .92 \\
\hline CEAS: Compassion from Others (10-100) & $52.4(22.0) ; 10-96$ & .96 \\
\hline MASS (1-6) & $3.6(1.0) ; 1-6$ & .91 \\
\hline DASS-21: Depression (0-42) & $18.0(13.0) ; 0-42$ & .94 \\
\hline DASS-21: Anxiety (0-42) & $10.5(9.7) ; 0-42$ & .85 \\
\hline DASS-21: Stress (0-42) & $20.2(10.4) ; 0-42$ & .88 \\
\hline Brief COPE: Emotion-focused coping (10-40) & $22.9(5.0) ; 13-36$ & .70 \\
\hline Brief COPE: Problem-focused coping (6-24) & $15.6(4.1) ; 6-24$ & .82 \\
\hline DERS (36-180) & $88.8(25.6) ; 44-178$ & .95 \\
\hline $\begin{array}{l}\text { Note. } \text { CEAS = Compassionate Engagement and } \\
\text { Awareness Scale: DASS-21 = Depression, Anxi } \\
\text { in Emotion Regulation Scale } \\
n=141\end{array}$ & $\begin{array}{l}\text { n Scales; MAAS = } \\
\text { nd Stress Scale-21; }\end{array}$ & $\begin{array}{l}\text { indful Attention } \\
\text { ERS }=\text { Difficulties }\end{array}$ \\
\hline
\end{tabular}


Table 3. Frequencies of categories for perceived severity of symptoms for depression, anxiety, and stress

\begin{tabular}{llll}
\hline Categories & Depression & Anxiety & Stress \\
\cline { 2 - 4 } & $n(\%)$ & $n(\%)$ & $n(\%)$ \\
\hline Normal & $42(29.8)$ & $36(25.5)$ & $66(46.8)$ \\
Mild & $14(9.9)$ & $6(4.3)$ & $18(12.8)$ \\
Moderate & $37(26.2)$ & $24(17.0)$ & $17(12.1)$ \\
Severe & $11(7.8)$ & $18(12.8)$ & $29(20.6)$ \\
Extremely severe & $37(26.2)$ & $57(40.4)$ &
\end{tabular}

Note. Manualised cut-off scores, as defined by Lovibond and Lovibond (1995). Depression: normal $=0-9 ;$ mild $=10-23 ;$ moderate $=14-20 ;$ severe $=21-27$; extremely severe $=28+$. Anxiety: normal $=0-7 ;$ mild $=8-9 ;$ moderate $=10-14$; severe $=15-19$; extremely severe $=$ $20+$. Stress: normal $=0-14 ;$ mild $=15-18 ;$ moderate $=19-25$; severe $=26-33$; extremely severe $=34+$ $n=141$ 
Table 4. Bivariate correlation matrix of study variables

\begin{tabular}{|c|c|c|c|c|c|c|c|c|c|c|}
\hline & & 1 & 2 & 3 & 4 & 5 & 6 & 7 & 8 & 9 \\
\hline 1. & CEAS: Compassion for Self & - & & & & & & & & \\
\hline 2. & CEAS: Compassion for Others & $.19^{*}$ & - & & & & & & & \\
\hline 3. & CEAS: Compassion from Others & $.44^{* *}$ & .03 & - & & & & & & \\
\hline 4. & DASS-21: Depression & $-.46 * *$ & $.17 *$ & $-.39 * *$ & - & & & & & \\
\hline 5. & DASS-21: Anxiety & $-.34 * *$ & $.18^{*}$ & $-.19 *$ & $.62 * *$ & - & & & & \\
\hline 6. & DASS-21: Stress & $-.38 * *$ & $.19 *$ & $-.22 *$ & $.72 * *$ & $.75^{* *}$ & - & & & \\
\hline 7. & MASS & $.36^{* *}$ & $-.21 *$ & .06 & $-.48 * *$ & $-.50 * *$ & $-.58 * *$ & - & & \\
\hline 8. & COPE Brief: Emotion-focused coping & $.38 * *$ & .06 & $.36^{* *}$ & $-.35^{* *}$ & -.08 & $-.19 *$ & .15 & - & \\
\hline 9. & COPE Brief: Problem-focused coping & $.46^{* *}$ & .05 & $.46^{* *}$ & $-.34 * *$ & -.16 & $-.24 * *$ & $.21 *$ & $.49 * *$ & - \\
\hline 10. & DERS & $-.59 * *$ & .08 & $-.30 * *$ & $.69 * *$ & $.59 * *$ & $.70 * *$ & $-.58 * *$ & $-.32 * *$ & $-.27 * *$ \\
\hline
\end{tabular}

Note. CEAS = Compassionate Engagement and Action Scales; DASS-21 = Depression, Anxiety, and Stress Scale-21; MAAS = Mindful Attention Awareness Scale; DERS $=$ Difficulties in Emotion Regulation Scale $n=141$

$* * p<.01$

$* p<.05$ 


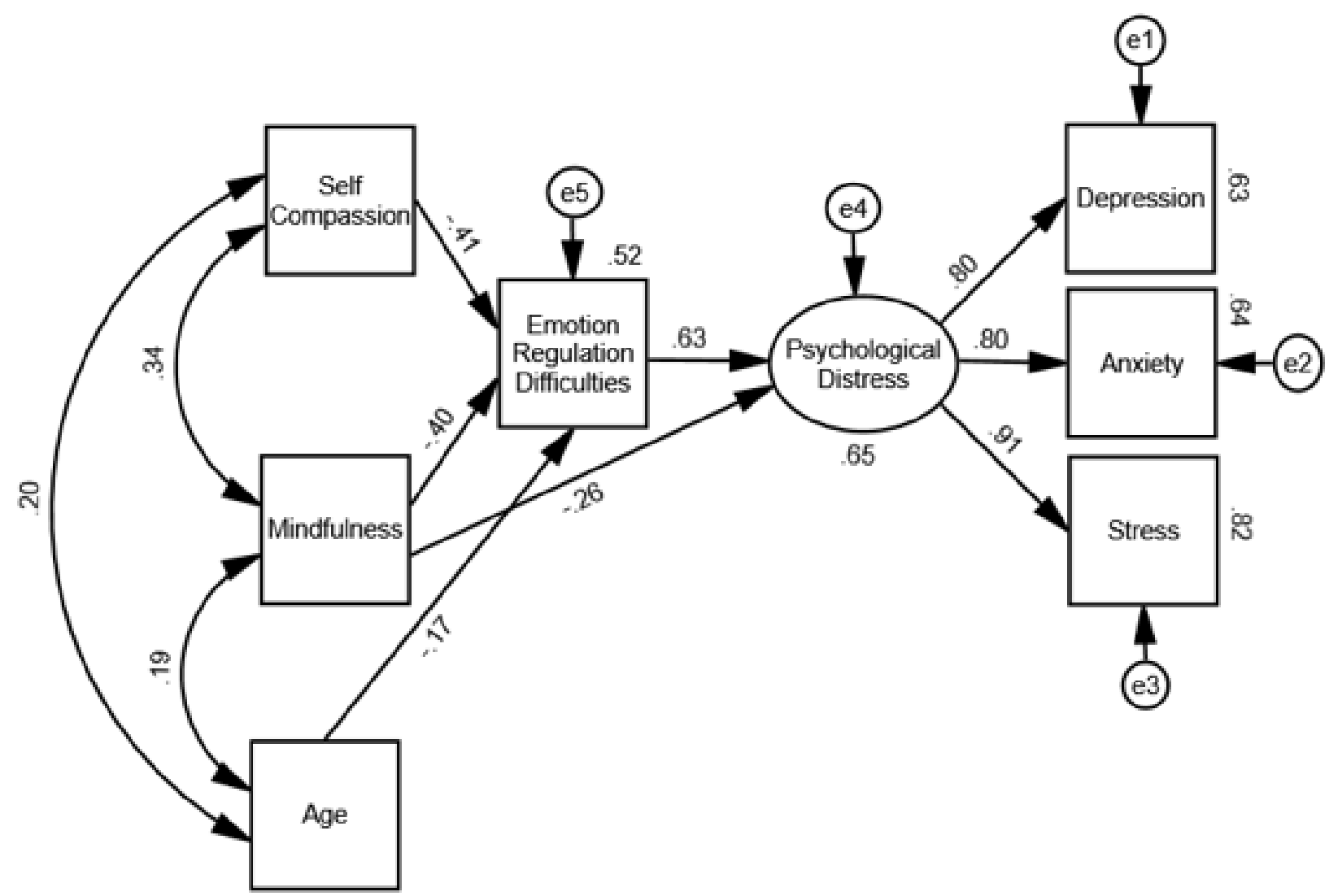

Figure 1. Standardised parameter estimates from the path analysis $(n=139)$ showing the relationships between self-compassion, dispositional mindfulness, difficulties in emotion regulation, and psychological distress after controlling for age of the family carer 


\section{Supplementary Data (Online Only)}

Table 5. Standardized and unstandardized parameter estimates from the path analysis $(n=139)$

\begin{tabular}{|c|c|c|c|c|}
\hline \multirow[t]{2}{*}{ Model pathways } & \multirow{2}{*}{$\begin{array}{l}\text { Standardized } \\
\beta\end{array}$} & \multicolumn{2}{|c|}{ Unstandardized } & \multirow{2}{*}{$\begin{array}{l}\text { Squared multiple correlations } \\
R^{2}\end{array}$} \\
\hline & & $B$ & $S E$ & \\
\hline Difficulties in emotion regulation & & & & .52 \\
\hline Difficulties in emotion regulation $\leftarrow$ Self-compassion & -.41 & $-.67 * * *$ & .10 & \\
\hline Difficulties in emotion regulation $\leftarrow$ Dispositional mindfulness & -.40 & $-10.47 * * *$ & 1.66 & \\
\hline Difficulties in emotion regulation $\leftarrow$ Age of the carer & -.17 & $-.37 * *$ & .13 & \\
\hline Psychological distress & & & & .65 \\
\hline Psychological distress $\leftarrow$ Dispositional mindfulness & -.26 & $-2.52 * * *$ & .70 & \\
\hline Psychological distress $\leftarrow$ Difficulties in emotion regulation & .63 & $.23 * * *$ & .03 & \\
\hline Depression & & & & .63 \\
\hline Depression $\leftarrow$ Psychological distress & .80 & $1.10 * * *$ & .09 & \\
\hline Anxiety & & & & .64 \\
\hline Anxiety $\leftarrow$ Psychological distress & .80 & $.83 * * *$ & .07 & \\
\hline Stress & & & & .82 \\
\hline Stress $\leftarrow$ Psychological distress & .91 & $1.00^{\mathrm{a}}$ & & \\
\hline
\end{tabular}

